\title{
Métodos alternativos para o ensino da anatomia humana: revisão sistematizada
}

\author{
Alternative methods for human anatomy teaching: systematic review \\ Métodos alternativos para la enseñanza de la anatomía humana: revisión sistematizada
}

Recebido: 20/11/2021 | Revisado: 29/11/2021 | Aceito: 03/12/2021 | Publicado: 14/12/2021

\author{
Diego Pires Rocha \\ ORCID: https://orcid.org/0000-0002-5124-0077 \\ Universidade de Pernambuco, Brasil \\ E-mail: diego.rocha@upe.br \\ Kleiton Giliarde Almeida da Silva \\ ORCID: https://orcid.org/0000-0001-9425-0331 \\ Universidade de Pernambuco, Brasil \\ kleitongalmeidas@gmail.com \\ Iracema Hermes Pires de Mélo Montenegro \\ ORCID: https://orcid.org/0000-0003-2770-8060 \\ Universidade de Pernambuco, Brasil \\ E-mail: iracema.montenegro@upe.br \\ Paulo Adriano Schwingel \\ ORCID: https://orcid.org/0000-0002-2935-3403 \\ Universidade de Pernambuco, Brasil \\ E-mail: paulo.schwingel@upe.br
}

\begin{abstract}
Resumo
As metodologias alternativas para o ensino-aprendizagem têm se destacado no ensino da anatomia, pois, permite ao aluno um papel ativo no processo de aprendizagem o que é a procura do ensino por descoberta, buscando assim superar as limitações do ensino. Visando revisar sistematicamente na literatura os métodos didáticos mais utilizados na anatomia, foi realizada uma busca nas bases de dados PubMed. Foram incluídos artigos originais que trabalharam a anatomia humana no ensino superior presencial, desde que tratassem o processo ensino-aprendizagem utilizando modelos educacionais alternativos. Foram encontrados 180 artigos no cruzamento dos seguintes descritores: "human anatomy", "higher education", "teaching" e "educational models"; dos quais 26 deles foram selecionados. Os estudos analisados demostraram a falta de continuidade nas pesquisas sobre a utilização de métodos alternativos no ensino de anatomia, e que a maioria desses trabalhos publicados são de instituições internacionais. Dos métodos de ensino citados nos estudos, foram variados envolvendo em sua maioria, as metodologias ativas. Os métodos de ensino de dissecação cadavérica e impressões 3D se destacaram quando comparadas com outras alternativas de ensino. Diante disso, os estudos identificados sobre metodologias alternativas para o ensino da anatomia humana indicam a eficiência desses métodos por estimularem a satisfação do discente pela disciplina e pelo aprendizado significativo.
\end{abstract}

Palavras-chave: Revisão sistemática; Anatomia; Ensino superior; Metodologias alternativas.

\begin{abstract}
Alternative methodologies for teaching and learning have stood out in the teaching of anatomy. Because it allows the student to take an active role in the learning process, which is what education seeks by discovery, thus seeking to overcome the limitations of teaching. To systematically review in the literature, the teaching methods most used in anatomy, a search was performed in the PubMed databases. Original articles that worked on human anatomy in faceto-face higher education were included, if they dealt with the teaching-learning process using alternative educational models. 180 articles were found at the intersection of the following descriptors: "human anatomy", "higher education", "teaching" and "educational models"; of which 26 were selected. The analyzed studies demonstrated the lack of continuity in research on the use of alternative methods in teaching anatomy, and that most of these published works are from international institutions. Of the teaching methods cited in the studies, they were varied, mostly involving the active methodologies. The teaching methods of cadaveric dissection and 3D impressions stood out when compared to other teaching alternatives. Therefore, the studies identified on alternative methodologies for teaching human anatomy indicate the efficiency of these methods as they stimulate student satisfaction with the discipline and significant learning.
\end{abstract}

Keywords: Systematic review; Anatomy; University education; Alternative methodologies.

\section{Resumen}

En la enseñanza de la anatomía se han destacado las metodologías alternativas de enseñanza-aprendizaje, ya que permiten que el alumno tenga un papel activo en dicho proceso, es decir el de una búsqueda de la enseñanza por descubrimiento, con la intención de superar las limitaciones de la enseñanza. Con el objetivo de revisar sistemáticamente en la literatura los métodos didácticos más utilizados en anatomía, se realizó una investigación en 
las bases de datos de PubMed. Se incluyeron artículos originales que trabajaban sobre la anatomía humana en la enseñanza presencial superior, siempre que trataran del proceso de enseñanza-aprendizaje mediante modelos educativos alternativos. Se han encontrado 180 artículos en el cruce de los siguientes términos: "human anatomy", "higher education", "teaching" y "educational models"; de los cuales 26 han sido seleccionados. Los estudios analizados demostraron una falta de continuidad en la investigación sobre el uso de métodos alternativos en la enseñanza de la anatomía, así como que la mayoría de estos trabajos publicados provienen de instituciones internacionales. Se encontraron varios métodos de enseñanza entre los citados en los estudios; en su mayoría métodos relacionados con las metodologías activas. Los métodos de enseñanza de disección cadavérica e impresiones en 3D destacaron en comparación con otras alternativas de enseñanza. En vista de ello, los estudios identificados sobre metodologías alternativas para la enseñanza de la anatomía humana indican la eficacia de estos métodos al estimular la satisfacción del estudiante en relación con la disciplina y con el aprendizaje significativo.

Palabras clave: Revisión sistemática; Anatomía; Educación superior; Metodologías alternativas.

\section{Introdução}

Anatomia é uma das ciências médicas mais antigas e se dedica ao estudo das estruturas e organização geral dos seres vivos (Salbego \& Oliveira, 2015; Costa \& Costa, 2012). Atualmente, o estudo anatômico na maioria das instituições do ensino superior é realizado por meio de uma combinação de aulas teóricas, estudo autodirigido com livros didáticos e aulas práticas em laboratório (Mclachlan \& Bligh, 2004). Esses métodos tradicionais de ensino na anatomia são bastante utilizados por muitas instituições de ensino superior (Mclachlan \& Patten, 2006; Drake \& Mcbride, 2009). Porém, devido à memorização breve, eles dificultam a retenção do conhecimento anatômico e, talvez, por isso, os estudantes relatam ser de difícil aprendizagem (Mansini \& Moreira, 2008; Brinke \& Klitsie, 2014).

De forma prática, a maioria dos docentes preferem utilizar a dissecação cadavérica como método de ensino (Patel \& Moxham, 2005). Porém, tanto a escassez quanto a burocracia na obtenção de cadáveres e as dificuldades que os estudantes têm para utilizar esse material para estudar, torna muitas vezes um fator limitante e desmotivador. Além disso, esses materiais acabam sendo disponibilizados aos estudantes apenas durante o horário da aula, fato que impede a utilização pelo tempo necessário ao estudo desta ciência (Inzunza \& D'acuña, 2003). Destaca-se ainda que a escassez de peças cadavéricas termina exigindo que o professor reflita sobre o processo ensino-aprendizagem e inove sua prática pedagógica oferecendo meios para integrar, aperfeiçoar e dinamizar as aulas de anatomia (Fornaziero \& Gordan, 2010).

Uma possibilidade de promover a retenção do conhecimento e amenizar a falta de material cadavérico necessário para o processo ensino-aprendizagem é utilizar metodologias alternativas que possam permitir uma maior participação e apreensão dos conhecimentos anatômicos (Johnson \& Charchanti, 2012). Neste sentido, as metodologias alternativas têm se destacado no ensino da anatomia por permitir ao aluno um papel ativo no processo de aprendizagem, proporcionando o ensino por descoberta e buscando superar as limitações do ensino tradicional.

No ensino da anatomia, a utilização de imagens, mesmo de produção amadora, contribui para alcançar satisfatoriamente os objetivos propostos, fato que melhora significativamente a compreensão dos alunos a respeito do conteúdo anatômico abordado e permite a eles a criação de jogos didáticos (Silva \& Santana, 2012). Neste ínterim, atividades lúdicas e/ou educativas podem se tornar uma atividade prazerosa ao mesmo tempo que favorecem a aprendizagem e permitem que o conhecimento do aluno seja mais completo (Soares, 2008). A utilização dessas práticas pedagógicas no ensino de anatomia representa uma possibilidade adicional aos professores, uma vez que estimula o aprendizado, de modo que os participantes desse processo passem a investigar as soluções para os problemas e para as situações em estudo. Essa nova maneira de ensinar está relacionada a uma nova visão de construção do conhecimento, em um processo que envolve todos os participantes, professores e alunos, superando as formas tradicionais na relação ensino-aprendizagem (Garcia, 2013).

Diante do exposto, no tocante a necessidade da utilização de diferentes técnicas de ensino a fim de favorecer uma aprendizagem eficiente, foram pesquisados estudos envolvendo metodologias alternativas para o ensino da anatomia humana 
no ensino superior.

\section{Metodologia}

Dois autores/avaliadores realizam busca eletrônica na base de dados PubMed por trabalhos publicados até março de 2020. Os descritores utilizados para a revisão sistemática foram selecionados mediante consulta ao Descritores em Ciências da Saúde (DeCS). Para o rastreamento das publicações, os descritores utilizados foram na língua inglesa: "human anatomy", "higher education", "teaching" e "educational models" associados ao operador lógico "AND”. A pesquisa não contou com restrição de idiomas, nem quanto ao período de publicação, sendo os artigos selecionados posteriormente por critérios de inclusão e exclusão. A qualidade metodológica de todos os estudos foi avaliada de forma independente por dois revisores.

Por meio deste procedimento de busca, foram identificadas 180 publicações potencialmente elegíveis para inclusão nesta revisão. Essas publicações apresentavam o resumo da obra disponível, o qual foi analisado quanto ao seguinte critério de inclusão: utilização de metodologias alternativas no processo ensino-aprendizagem da disciplina de anatomia humana para estudantes de nível superior. As publicações selecionadas foram avaliadas em relação os seguintes critérios de exclusão: (a) artigos de revisão; (b) aplicação da pesquisa em outros níveis de ensino; (c) metodologia de ensino tradicional; (d) utilização de aulas teóricas; (e) outra população de estudo que seja de discentes.

Figura 1. Esquema da seleção dos artigos.

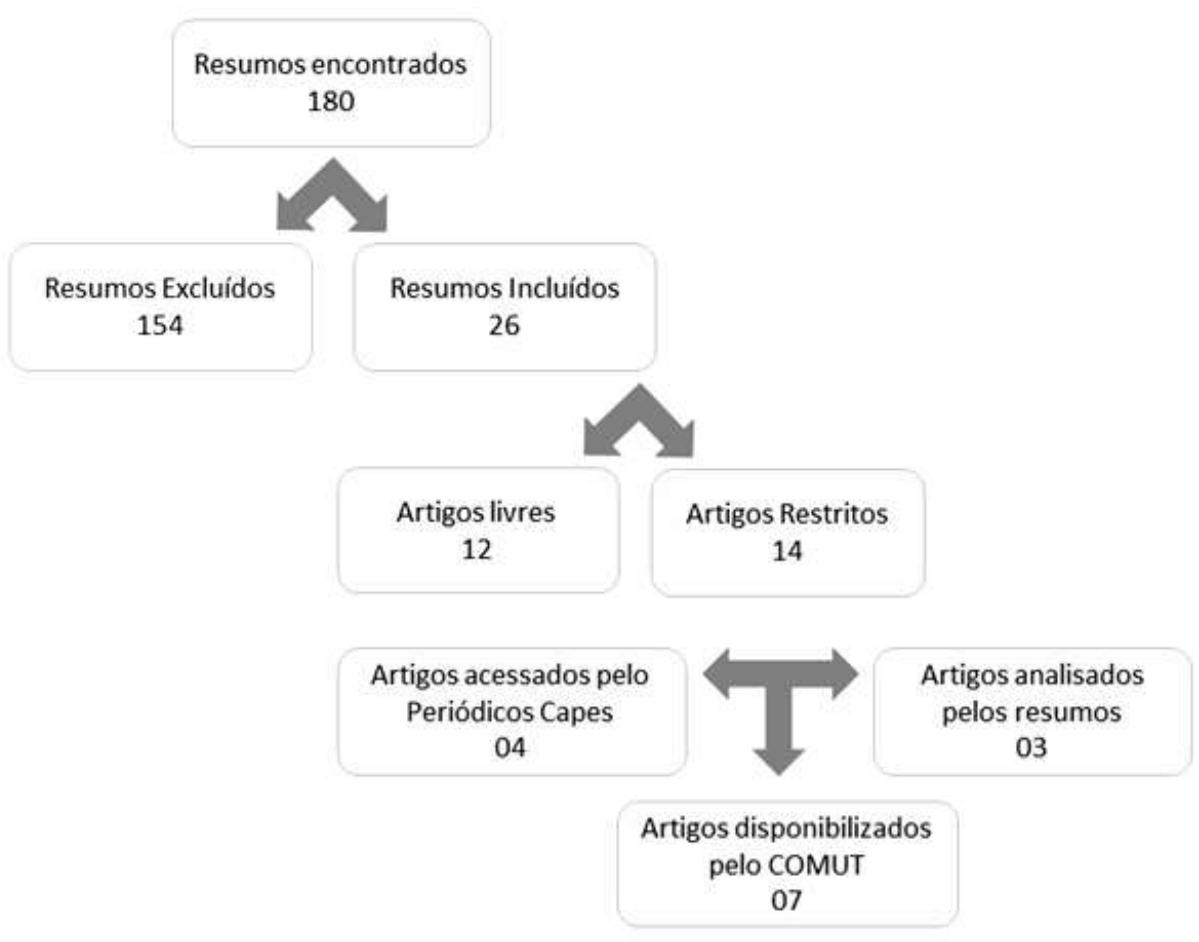

Fonte: Autores.

Ao fim dessa primeira etapa, foram identificadas 26 publicações para serem analisadas. A segunda etapa constou da busca e posterior avaliação na íntegra desses artigos. Dos 26 resumos selecionados para análise, apenas doze estavam disponíveis por completo na base de dados pesquisada. As outras 14 publicações com resumo disponível tiveram seus artigos completos pesquisados no Portal de Periódicos da CAPES mediante assinatura da Universidade de Pernambuco (UPE), sendo encontrado quatro artigos completos. Dos 10 artigos restantes, 07 foram adquiridos por meio do Programa de Comutação Bibliográfica (COMUT) e os três artigos restantes foram analisados apenas pelos resumos disponibilizados. 
Após a avaliação dos artigos completos, cinco entraram nos critérios de exclusão não identificados anteriormente no resumo disponível, pelos seguintes motivos a seguir descritos: (a) opinião discente sobre as metodologias aplicadas normalmente nas disciplinas existentes foi avaliada e não um método específico; (b) o local de estudo pertencia ao nível básico de educação; (c) a opinião docente sobre as metodologias foi avaliada e não um método específico; (d) não avaliou um método de ensino sendo aplicado apenas um questionário para saber quais os métodos utilizados; (e) a disciplina avaliada foi a de patologia humana.

Ao finalizar as análises, a base de dados desse estudo constou de 26 artigos selecionados, que foram organizados e apresentados cronologicamente em dois quadros. O primeiro quadro caracteriza os artigos quanto ao autor/ano, revista, instituição do primeiro autor e disponibilidade do artigo. O segundo quadro avalia o objetivo desse estudo, sendo exibido os dados: população estudada; local de estudo; métodos de ensino utilizados na anatomia e resultados encontrados. O método de ensino foi avaliado quanto: independente ou em grupo; passivo, ativo ou passivo/ativo; estratégia metodológica sendo única ou mais de uma.

$\mathrm{O}$ método independente consiste em atividades diversas orientadas pelo professor, de forma clara, objetiva e sistematizada, permitindo que os alunos realizem o trabalho de forma independente e criativa. Já o método em grupo, consiste na aprendizagem a partir da cooperação dos alunos entre si, de modo que haja a troca de saberes e experiências (Libânio, 2013). O método ativo ocorre quando o aluno interage com o assunto em estudo, sendo estimulado a construir o conhecimento ao invés de recebê-lo do professor com o método passivo (Barbosa \& Moura, 2013). A estratégia metodológica retrata o elemento da didática na qual o professor organiza as atividades de ensino para que os alunos possam atingir objetivos em relação a um conteúdo específico (Severino, 2009).

\section{Resultados e Discussão}

Os estudos sobre metodologias de ensino com utilização de modelos educacionais alternativos aplicadas à disciplina de anatomia humana para o nível superior apresentados na base de dados PubMed, iniciaram suas publicações em 2001, seguindo até 2019. Apesar de iniciarem em 2001, apenas a partir do ano de 2008 elas ocorreram sem interrupções até 2015, quando novamente se observa ausência de estudos em 2016, seguindo depois, de 2017 a 2019. A maioria das publicações ocorreu em $2014(n=04 ; 15,4 \%)$ e em $2015(n=04 ; 15,4 \%)$, seguidos de $2010(n=03 ; 11,5 \%), 2011(n=03 ; 11,5 \%)$ e 2012 $(\mathrm{n}=03 ; 11,5 \%)$. O quadro 1 demonstra que os pesquisadores realizam estudos pontuais sobre o processo ensino-aprendizagem da anatomia humana na educação superior, especificamente sobre metodologias alternativas.

Destaca-se a importância de pesquisas continuadas envolvendo o ensino da anatomia, visto que, o método tradicional (palestras e dissecação cadavérica) incorpora nessa ciência uma memorização interminável, desestimulando o aprendizado e que, as atividades lúdico-educativas favorecem à aprendizagem, permitindo que a aquisição do conhecimento pelo discente seja mais prazeroso (Miller, et al., 2002; Soares, 2008).

As instituições que mais publicam sobre o tema em questão são do exterior $(n=19 ; 73,1 \%)$. Entre os países, os Estados Unidos da América predominam em publicações sobre o tema $(n=10 ; 38,5 \%)$, seguido de diversos países Europeus $(\mathrm{n}=07 ; 26,9 \%)$ e do Brasil $(\mathrm{n}=04 ; 15,4 \%)$. Apesar da grande variabilidade das instituições que estudam o tema, destacam-se a Universidade de Maastricht $(\mathrm{n}=02 ; 7,7 \%)$ e a Universidade de Londres $(\mathrm{n}=02 ; 7,7 \%)$. As pesquisas foram publicadas tanto em periódicos internacionais $(n=19 ; 73,1 \%)$ quanto nacionais $(n=04 ; 15,4 \%)$. Os estudos internacionais apresentaram predominância de publicação na Anatomical Science Education $(n=11 ; 42,3 \%)$. Nas pesquisas nacionais, houve diversidade de periódicos para publicação. Apenas 30,8\% $(\mathrm{n}=08)$ dos artigos se encontravam com acesso aberto, inclusive corresponderam a todas as publicações nacionais (Quadro 1). A escassez de publicações nacionais pode possivelmente estar 
associada à formação docente. Em geral, professores brasileiros de anatomia concluíram sua graduação em cursos de bacharelado, e por não serem licenciados sentem dificuldade em realizar pesquisas em educação.

Quadro 1. Estudos sobre metodologias de ensino com utilização de modelos educacionais alternativos aplicadas à disciplina de Anatomia Humana para o nível superior identificado por autor/ano, revista de publicação, instituição do primeiro autor e disponibilidade do artigo completo.

\begin{tabular}{|c|c|c|c|}
\hline Autor, ano & Nome do periódico & Instituição do primeiro autor & $\begin{array}{l}\text { Disponibilidade do } \\
\text { artigo completo }\end{array}$ \\
\hline Silva \& Silva, 2001 & Arquivos da APADEC & Universidade Federal de Goiás, Brasil & Livre \\
\hline Montes \& Souza, 2008 & $\begin{array}{c}\text { Alexandria: Revista de Educação } \\
\text { em Ciência e Tecnologia }\end{array}$ & Universidade Gama Filho, Brasil & Livre \\
\hline Khalil et al., 2008 & Anatomical Science Education & $\begin{array}{c}\text { Tuskegee University, Estados Unidos } \\
\text { da América (EUA) }\end{array}$ & Resumo \\
\hline Gookin \& Foster, 2009 & $\begin{array}{c}\text { Journal of Veterinary Medical } \\
\text { Education }\end{array}$ & North Carolina State University, EUA & Resumo \\
\hline Silva \& Aguiar Júnior, 2010 & Congresso Internacional PBL 2010 & $\begin{array}{c}\text { Universidade Federal de São Paulo, } \\
\text { Brasil }\end{array}$ & Livre \\
\hline Montes \& Souza, 2010 & Ciências \& Cognição & Fundação Oswaldo Cruz, Brasil & Livre \\
\hline Estevez \& Lindgren, 2010 & Anatomical Science Education & University of Massachusetts, EUA & Livre \\
\hline Codd \& Choudhury, 2011 & Anatomical Science Education & Durham University, Reino Unido & COMUT \\
\hline Keedy et al., 2011 & Anatomical Science Education & University of California, EUA & Resumo \\
\hline Waters \& Meter, 2011 & Advances in Physiology Education & Pennsylvania State University, EUA & Livre \\
\hline Braid \& Williams, 2012 & Anatomical Science Education & University of London, Reino Unido & COMUT \\
\hline Ruisoto \& Juanes, 2012 & Anatomical Science Education & Universidad de Salamanca, Espanha & COMUT \\
\hline Knobe \& Carow, 2012 & BMC Medical Education & $\begin{array}{c}\text { Rheinisch-Westfälische Technische } \\
\text { Hochschule Aachen, Alemanha }\end{array}$ & Livre \\
\hline Preece \& Williams, 2013 & Anatomical Science Education & University of London, Reino Unido & COMUT \\
\hline Bergman et al., 2013 & Anatomical Science Education & Maastricht University, Holanda & COMUT \\
\hline Kooloos \& Schepens-Franke, 2014 & Anatomical Science Education & Radboud Universiteit, Holanda & COMUT \\
\hline Serrat \& Dom, 2014 & Anatomical Science Education & Marshall University, EUA & COMUT \\
\hline Haspel \& Motoike, 2014 & Anatomical Science Education & City University of New York, EUA & Livre \\
\hline Lombardi \& Hicks, 2014 & Advances in Physiology Education & University of Maryland, EUA & Livre \\
\hline Gonsalvezet al., 2015 & BMC Medical Education & University of Melbourne, Austrália & Portal de Periódicos \\
\hline Han et al., 2015 & PLOS ONE & $\begin{array}{c}\text { Chonnam National University } \\
\text { Hospital, Coréia do Sul }\end{array}$ & Portal de Periódicos \\
\hline Bergman et al., 2015 & BMC Medical Education & Maastricht University, Holanda & Livre \\
\hline Li \& Li, 2015 & Scientific Reports & Binzhou Medical University, China & Livre \\
\hline Jones \& Seckeler, 2017 & Congenital Heart Disease & University of Arizona, EUA & Portal de Periódicos \\
\hline
\end{tabular}




\begin{tabular}{l|c|c|c}
\hline Akle et al., 2018 & Anatomical Science Education & Universidad de los Andes, Colômbia & Portal de Periódicos \\
\hline Ladage et al., 2018 & Advances in Physiology Education & Pennsylvania State University, EUA & Livre \\
\hline
\end{tabular}

Fonte: Autores.

A metodologia dos estudos quanto ao local e população de estudo não foi uniforme. O local de estudo foi semelhante à instituição de vínculo do primeiro autor, sendo a maioria de três locais: Maastricht University $(\mathrm{n}=2 ; 7,7 \%)$, Pennsylvania State University $(\mathrm{n}=2 ; 7,7 \%)$ e University of London $(\mathrm{n}=2 ; 7,7 \%)$. Apenas um estudo foi multicêntrico, pois estudou a metodologia de ensino em anatomia humana em diversas universidades da Espanha (Madrid, Salamanca, Valladolid, Pamplona e Barcelona). A população de estudo prioritária foi de diversos cursos da área da saúde ( $\mathrm{n}=10 ; 38,5 \%)$, seguido de medicina $(\mathrm{n}=05 ; 19,2 \%)$ e medicina veterinária $(\mathrm{n}=02 ; 7,7 \%)$ (Quadro 2).

Quadro 2. Apresentação dos estudos sobre metodologias de ensino com utilização de modelos educacionais alternativos aplicadas à disciplina de Anatomia Humana para o nível superior identificado por autor/ano, local de estudo, população de estudo, método de ensino e resultados.

\begin{tabular}{|c|c|c|c|c|}
\hline Autor, ano & Local do estudo & $\begin{array}{l}\text { População do } \\
\text { estudo }\end{array}$ & Método de ensino & Resultados do método de ensino \\
\hline $\begin{array}{l}\text { Silva \& Silva, } \\
2001\end{array}$ & $\begin{array}{l}\text { Universidade Federal } \\
\text { de Goiás, Brasil }\end{array}$ & $\begin{array}{l}30 \text { estudantes de } \\
\text { licenciatura em } \\
\text { ciências biológicas }\end{array}$ & $\begin{array}{l}\text { Artes cênicas: desenvolvimento } \\
\text { de peças teatrais. }\end{array}$ & $\begin{array}{l}\text { As peças teatrais associadas à ciência } \\
\text { possibilitam a construção ativa do } \\
\text { conhecimento. }\end{array}$ \\
\hline $\begin{array}{l}\text { Montes \& } \\
\text { Souza, } 2008\end{array}$ & $\begin{array}{l}\text { Campus da Fundação } \\
\text { Oswaldo Cruz, Brasil }\end{array}$ & $\begin{array}{l}20 \text { estudantes de } \\
\text { fisioterapia e } \\
\text { educação física }\end{array}$ & $\begin{array}{l}\text { Ensino à população através de } \\
\text { Exposição de Peças } \\
\text { Anatômicas. }\end{array}$ & $\begin{array}{l}\text { Os discentes participam como } \\
\text { educadores em eventos não formais de } \\
\text { ensino, valorizando e estimulando } \\
\text { práticas de ensino mais dinâmicas } \\
\text { contribuindo para um aprendizado } \\
\text { significativo. }\end{array}$ \\
\hline $\begin{array}{l}\text { Khalil et al., } \\
2008\end{array}$ & $\begin{array}{l}\text { Universidade do } \\
\text { Estado da Flórida, } \\
\text { Tallahassee, Florida. }\end{array}$ & $\begin{array}{l}28 \text { calouros de } \\
\text { medicina e } 208 \\
\text { estudantes de biologia }\end{array}$ & $\begin{array}{l}\text { Uso de três estratégias de } \\
\text { instruções baseadas em } \\
\text { computador afim de melhorar a } \\
\text { interpretação de imagens } \\
\text { radiológicas. }\end{array}$ & $\begin{array}{l}\text { O estudo mostrou que no ambiente } \\
\text { baseado em computadores } \\
\text { autodirigidos não houve efeitos } \\
\text { positivos significativos. }\end{array}$ \\
\hline $\begin{array}{l}\text { Gookin \& } \\
\text { Foster, } 2009\end{array}$ & $\begin{array}{l}\text { Universidade Estadual } \\
\text { da Carolina do Norte, } \\
\text { Estados Unidos da } \\
\text { América (EUA) }\end{array}$ & $\begin{array}{l}74 \text { alunos do primeiro } \\
\text { ano de veterinária }\end{array}$ & $\begin{array}{l}\text { Uso de modelo animado para } \\
\text { ensinar motilidade } \\
\text { reticulorumênica. }\end{array}$ & $\begin{array}{l}\text { Os alunos de veterinária foram } \\
\text { colocados de forma aleatória em dois } \\
\text { grupos de estudos: grupo A, com o } \\
\text { modelo de ensino animado; e o grupo } \\
\text { B, com textos e desenhos. Os } \\
\text { resultados mostraram que a maioria } \\
\text { dos alunos preferiram o uso do } \\
\text { modelo animado. }\end{array}$ \\
\hline $\begin{array}{l}\text { Silva \& Aguiar } \\
\text { Júnior, } 2010\end{array}$ & $\begin{array}{l}\text { Universidade Federal } \\
\text { de São Paulo, Brasil }\end{array}$ & $\begin{array}{c}\text { Estudantes de } \\
\text { educação física, } \\
\text { fisioterapia, nutrição, } \\
\text { psicologia e terapia } \\
\text { ocupacional }\end{array}$ & $\begin{array}{l}\text { Desenvolvimento de um projeto } \\
\text { de pesquisa na área profissional } \\
\text { e relacionado à disciplina } \\
\text { utilizando construção de } \\
\text { modelos tridimensionais, } \\
\text { recursos tecnológicos ou } \\
\text { dissecação. }\end{array}$ & $\begin{array}{l}\text { Modelos tridimensionais auxiliam na } \\
\text { criatividade e aprendizagem } \\
\text { significativa. Os melhores resultados } \\
\text { foram verificados na dissecação } \\
\text { seguido dos modelos tecnológicos. }\end{array}$ \\
\hline $\begin{array}{l}\text { Montes \& } \\
\text { Souza, } 2010\end{array}$ & $\begin{array}{l}\text { Universidade Severino } \\
\text { Sombra, Brasil }\end{array}$ & $\begin{array}{l}\text { Estudantes da } \\
\text { graduação em } \\
\text { medicina }\end{array}$ & $\begin{array}{l}\text { Exposição teórica seguida de } \\
\text { abordagem prática com } \\
\text { dinâmicas propostas pelos } \\
\text { discentes (atlas digital, fotos de } \\
\text { patologias, relatórios, } \\
\text { seminários) pelos discentes. }\end{array}$ & $\begin{array}{l}\text { Os alunos aprendem por se tornar } \\
\text { atores de seu próprio aprendizado. } \\
\text { Isso motiva a aprenderem de forma } \\
\text { significativa, e de auxiliar o aluno a } \\
\text { assimilar a estrutura da matéria de } \\
\text { ensino e organizar a própria estrutura } \\
\text { cognitiva. }\end{array}$ \\
\hline $\begin{array}{l}\text { Estevez \& } \\
\text { Lindgren, } 2010\end{array}$ & $\begin{array}{l}\text { Escola de Medicina da } \\
\text { Universidade de } \\
\text { Boston, EUA }\end{array}$ & $\begin{array}{l}101 \text { estudantes do } \\
\text { primeiro ano de } \\
\text { medicina }\end{array}$ & $\begin{array}{l}\text { Avaliação de uma nova } \\
\text { ferramenta no ensino de } \\
\text { neuroanatomia 3D. }\end{array}$ & $\begin{array}{l}\text { Os alunos participantes do estudo } \\
\text { foram divididos de forma aleatória em } \\
\text { duas salas, uma experimental e outra } \\
\text { controle. Todos os alunos passaram }\end{array}$ \\
\hline
\end{tabular}




\begin{tabular}{|c|c|c|c|c|}
\hline & & & & $\begin{array}{l}\text { pelo método de ensino tradicional } \\
\text { (2D), em seguida, durante a revisão no } \\
\text { laboratório, os alunos do grupo } \\
\text { experimental construíram modelos } \\
\text { 3D, enquanto o grupo controle } \\
\text { reexaminou cortes do cérebro (2D). } \\
\text { Os resultados obtidos após avaliação } \\
\text { sugeriram que esse método é eficaz no } \\
\text { ensino de relações espaciais de } \\
\text { Anatomia. }\end{array}$ \\
\hline $\begin{array}{l}\text { Codd \& } \\
\text { Choudhury, } \\
2011\end{array}$ & $\begin{array}{l}\text { Universidade de } \\
\text { Manchester, Reino } \\
\text { Unido }\end{array}$ & $\begin{array}{l}\text { Alunos do módulo de } \\
\text { habilidades em } \\
\text { anatomia humana }\end{array}$ & $\begin{array}{l}\text { Criação modelos } \\
\text { tridimensionais em computador } \\
\text { através da realidade virtual e } \\
\text { dissecação. }\end{array}$ & $\begin{array}{l}\text { A aprendizagem da anatomia } \\
\text { utilizando a realidade virtual pode ser } \\
\text { usada para complementar o ensino } \\
\text { tradicional (dissecação). Os grupos de } \\
\text { dissecação e realidade virtual foram } \\
\text { semelhantes entre si e melhores que o } \\
\text { controle. }\end{array}$ \\
\hline $\begin{array}{l}\text { Keedy et al., } \\
2011\end{array}$ & $\begin{array}{l}\text { Universidade da } \\
\text { Califórnia, EUA }\end{array}$ & $\begin{array}{l}46 \text { estudantes de } \\
\text { medicina }\end{array}$ & $\begin{array}{l}\text { Avaliação da eficácia do uso de } \\
\text { apresentação tridimensional } \\
\text { interativa comparada com a } \\
\text { apresentação em formato de } \\
\text { livro. }\end{array}$ & $\begin{array}{l}\text { Os participantes do estudos foram } \\
\text { randomizados em dois grupos e } \\
\text { apresentados a um módulo de } \\
\text { aprendizado 3D ou 2D. Após cada } \\
\text { módulo os alunos responderam uma } \\
\text { pesquisa de satisfação e um pós-teste } \\
\text { de anatomia. O módulo 3D recebeu } \\
\text { classificação de satisfação mais alta } \\
\text { que o 2D, porém, esse método não } \\
\text { melhorou nem inibiu o aprendizado de } \\
\text { Anatomia. }\end{array}$ \\
\hline $\begin{array}{l}\text { Waters \& } \\
\text { Meter, } 2011\end{array}$ & $\begin{array}{l}\text { Universidade Estadual } \\
\text { da Pensilvânia, EUA }\end{array}$ & $\begin{array}{l}222 \text { alunos dos cursos } \\
\text { de enfermagem, } \\
\text { cinesiologia e outras } \\
\text { áreas da saúde }\end{array}$ & $\begin{array}{l}\text { Palestra inicial (Músculos). } \\
\text { Grupos: } \\
\text { - Controle: Dissecação em gatos } \\
\text { com folhetos informativos } \\
\text { (fotografias e diagramas de gato } \\
\text { e humano). } \\
\text { - Esculpir estruturas humanas } \\
\text { em argila com folhetos } \\
\text { informativos (fotografias e } \\
\text { diagramas de gato e humano) e } \\
\text { guia suplementar (Anatomia } \\
\text { funcional do sistema muscular). } \\
\text { - Dissecação em gatos com } \\
\text { folheto informativo e guia } \\
\text { suplementar (orientou a ordem } \\
\text { de dissecção e ação muscular) }\end{array}$ & $\begin{array}{l}\text { Os alunos esculpindo estruturas } \\
\text { humanas em argila obtiveram melhor } \\
\text { desempenho em comparação com os } \\
\text { alunos que realizam dissecções de } \\
\text { gato. Ou seja, é mais significativo o } \\
\text { aprendizado de músculos quanto } \\
\text { utilizado a espécie a ser estudada. }\end{array}$ \\
\hline $\begin{array}{l}\text { Braid \& } \\
\text { Williams, } 2012\end{array}$ & $\begin{array}{c}\text { Universidade de } \\
\text { Londres, Reino Unido }\end{array}$ & $\begin{array}{l}38 \text { estudantes do } \\
\text { curso de veterinária }\end{array}$ & $\begin{array}{l}\text { Uso de um tapete topográfico } \\
\text { de anatomia para equino } \\
\text { ("Anato-Rug") para ser usado } \\
\text { em um cavalo vivo. }\end{array}$ & $\begin{array}{l}\text { "Anato-Rug" é uma ferramenta de } \\
\text { aprendizado auxiliar na fixação do } \\
\text { conteúdo na memória a curto prazo; } \\
\text { assim como na compreensão, } \\
\text { confiança dos alunos e prazer em } \\
\text { aprender anatomia. Não notando } \\
\text { diferença na memória a longo prazo. }\end{array}$ \\
\hline $\begin{array}{l}\text { Ruisoto \& } \\
\text { Juanes, } 2012\end{array}$ & $\begin{array}{l}\text { Diversas universidades } \\
\text { da Espanha (Madrid, } \\
\text { Salamanca e } \\
\text { Valladolid, Pamplona e } \\
\text { Barcelona) } \\
\end{array}$ & $\begin{array}{l}80 \text { voluntários } \\
\text { psicólogos, médicos, } \\
\text { neuropsicólógos e } \\
\text { neuroanatomistas }\end{array}$ & $\begin{array}{l}\text { Imagens morfológicas em } 2 \mathrm{D} \text { e } \\
\text { 3D. }\end{array}$ & $\begin{array}{l}\text { Sistemas de visualização volumétrica } \\
\text { (3D) melhoram a educação em } \\
\text { neuroanatomia, especialmente, para } \\
\text { imagens difíceis de identificar. }\end{array}$ \\
\hline $\begin{array}{l}\text { Knobe \& } \\
\text { Carow, } 2012\end{array}$ & $\begin{array}{l}\text { Faculdade de } \\
\text { Medicina, Aachen, } \\
\text { Alemanha }\end{array}$ & $\begin{array}{l}\text { Estudantes de } \\
\text { medicina do segundo } \\
\text { ano }\end{array}$ & $\begin{array}{l}\text { Dissecação (controle); } \\
\text { ultrassom musculoesquelético } \\
\text { (MSUS); métodos } \\
\text { artroscópicos. }\end{array}$ & $\begin{array}{l}\text { Artroscopia para o treinamento de } \\
\text { anatomia melhorou a absorção do } \\
\text { conhecimento anatômico para regiões } \\
\text { complexas (ombro). O ultrassom } \\
\text { apesar de não melhorar a } \\
\text { aprendizagem auxiliou na didática } \\
\text { profissional. }\end{array}$ \\
\hline $\begin{array}{l}\text { Preece \& } \\
\text { Williams, } 2013\end{array}$ & $\begin{array}{c}\text { Universidade de } \\
\text { Londres, Reino Unido }\end{array}$ & $\begin{array}{l}\text { Os indivíduos foram } \\
\text { recrutados a partir do } \\
\text { terceiro ano do curso } \\
\text { de medicina }\end{array}$ & $\begin{array}{l}\text { Três grupos de ensino: livros } \\
\text { didáticos (atlas); modelo em } \\
\text { computador reconstruído em } \\
\text { 3D (imagem) e modelo físico }\end{array}$ & $\begin{array}{l}\text { O modelo plástico físico }(3 \mathrm{D}) \\
\text { demonstrou vantagens para melhorar a } \\
\text { apreciação visuoespacial e } \\
\text { compreensão da arquitetura anatômica }\end{array}$ \\
\hline
\end{tabular}


Research, Society and Development, v. 10, n. 16, e370101623641, 2021

(CC BY 4.0) | ISSN 2525-3409 | DOI: http://dx.doi.org/10.33448/rsd-v10i16.23641

\begin{tabular}{|c|c|c|c|c|}
\hline & & $\begin{array}{c}\text { veterinária na } \\
\text { instituição dos autores }\end{array}$ & da imagem em 3D. & complexa do pé equino. \\
\hline $\begin{array}{l}\text { Bergman et al., } \\
2013\end{array}$ & $\begin{array}{l}\text { Universidade de } \\
\text { Maastricht, Holanda }\end{array}$ & $\begin{array}{l}360 \text { estudantes de } \\
\text { medicina }\end{array}$ & $\begin{array}{l}\text { Utilizando o método PBL, um } \\
\text { manual foi construído para } \\
\text { auxiliar em práticas de } \\
\text { Anatomia de Superfície dentro } \\
\text { do contexto do exame físico } \\
\text { clínico. }\end{array}$ & $\begin{array}{l}\text { O ensino com exame físico utilizando } \\
\text { a anatomia de superfície de acordo } \\
\text { com o contexto construtivo, } \\
\text { colaborativo, contextual e com } \\
\text { princípios de aprendizagem } \\
\text { autodirigida leva à maior satisfação e } \\
\text { percepção de melhor aprendizado dos } \\
\text { alunos. }\end{array}$ \\
\hline $\begin{array}{l}\text { Kooloos \& } \\
\text { Schepens- } \\
\text { Franke, } 2014\end{array}$ & $\begin{array}{l}\text { Centro Médico da } \\
\text { Universidade Radboud, } \\
\text { Holanda }\end{array}$ & $\begin{array}{c}\text { Ensinou } \\
\text { simultaneamente para } \\
\text { estudantes de } \\
\text { primeiro ano de } \\
\text { medicina } \\
\text { (aproximadamente, } \\
\text { 340) e estudantes } \\
\text { biomédicos de } \\
\text { segundo ano } \\
\text { (aproximadamente, } \\
\text { 90). } 209 \text { alunos } \\
\text { realizaram exercício } \\
\text { de modelagem de } \\
\text { argila e } 190 \\
\text { visualizou o vídeo }\end{array}$ & $\begin{array}{l}\text { Dois grupos: } \\
\text { - Modelagem em argila de } \\
\text { estruturas anatômicas; } \\
\text { - Visualização em vídeo da } \\
\text { modelagem em argila. }\end{array}$ & $\begin{array}{l}\text { Os alunos que realizaram a } \\
\text { modelagem em argila apresentaram } \\
\text { melhor desempenho na retenção do } \\
\text { conhecimento anatômico que aqueles } \\
\text { que visualizaram o vídeo. Deve-se } \\
\text { incentivar os professores de anatomia } \\
\text { a desenvolver exercícios práticos que } \\
\text { ofereçam experiências de } \\
\text { aprendizagem que rastreiam a atenção } \\
\text { dos alunos. }\end{array}$ \\
\hline $\begin{array}{l}\text { Serrat \& Dom, } \\
2014\end{array}$ & $\begin{array}{l}\text { Universidade Marshall, } \\
\text { EUA }\end{array}$ & $\begin{array}{l}\text { O tamanho médio das } \\
\text { turmas nos últimos } \\
\text { cinco anos (2009- } \\
\text { 2013) foi de } 76 \text { alunos }\end{array}$ & $\begin{array}{l}\text { Palestra e depois formação de } \\
\text { dois grupos, baseada na } \\
\text { dissecação com ensino } \\
\text { (módulos de construções } \\
\text { tridimensionais para ajudar a } \\
\text { entender regiões anatômicas } \\
\text { complexas) e os demais } \\
\text { observaram. Depois o } \\
\text { observador torna-se dissecador. }\end{array}$ & $\begin{array}{l}\text { Os módulos de dissecação auxiliam a } \\
\text { aprendizagem por facilitar a } \\
\text { compreensão tridimensional do corpo } \\
\text { humano, podendo ser amplamente } \\
\text { adotado por ser de baixo custo. }\end{array}$ \\
\hline $\begin{array}{l}\text { Haspel \& } \\
\text { Motoike, } 2014\end{array}$ & $\begin{array}{l}\text { Faculdade Comunitária } \\
\text { LaGuardia, EUA }\end{array}$ & $\begin{array}{l}\text { Professores e alunos } \\
\text { do primeiro e segundo } \\
\text { semestre de anatomia } \\
\text { e fisiologia }\end{array}$ & $\begin{array}{l}\text { Transição do método de ensino } \\
\text { tradicional de Anatomia e } \\
\text { Fisiologia (dissecação de gatos) } \\
\text { para o uso de modelagem em } \\
\text { argila e ratos para dissecação. }\end{array}$ & $\begin{array}{l}\text { A maioria dos professores } \\
\text { consideraram esse método eficaz no } \\
\text { ensino. Para os alunos, esses métodos } \\
\text { os ajudaram a entender os assuntos } \\
\text { abordados. }\end{array}$ \\
\hline $\begin{array}{l}\text { Lombardi \& } \\
\text { Hicks, } 2014\end{array}$ & $\begin{array}{l}\text { Universidade de } \\
\text { Maryland, EUA }\end{array}$ & $\begin{array}{l}\text { Alunos inscritos na } \\
\text { anatomia humana e } \\
\text { fisiologia I de } \\
\text { diversos cursos da } \\
\text { universidade. } \\
\text { programas de } \\
\text { enfermagem, } \\
\text { programas de pós- } \\
\text { graduação e as } \\
\text { profissões médicas, } \\
\text { que pode exigir este } \\
\text { curso } \\
\end{array}$ & $\begin{array}{l}\text { Palestra ( } 15 \text { min) sobre o } \\
\text { sistema cardiovascular e } \\
\text { atividade }(45 \text { min }) \text { em três } \\
\text { grupos: dissecações de órgãos, } \\
\text { dissecações em mídias virtuais } \\
\text { ou modelos anatômicos de } \\
\text { plástico. }\end{array}$ & $\begin{array}{l}\text { Atividades diversas auxiliam as } \\
\text { diferentes formas de aprendizado. } \\
\text { Dissecações cadavéricas captam } \\
\text { melhor a essência da ciência, evitando } \\
\text { a simplificação excessiva promovida } \\
\text { pelos modelos de plástico ou } \\
\text { atividades virtuais. As dissecações de } \\
\text { órgãos tiveram maior valor percebido. } \\
\text { O uso de modelos plásticos pode } \\
\text { promover melhor aquisição e retenção } \\
\text { de conhecimento que as mídias } \\
\text { virtuais. }\end{array}$ \\
\hline $\begin{array}{l}\text { Gonsalvezet al., } \\
2015\end{array}$ & $\begin{array}{l}\text { Universidade de } \\
\text { Melbourne, Austrália }\end{array}$ & 450 alunos & $\begin{array}{l}\text { Aulas prática com dissecação } \\
\text { ou oficinas. }\end{array}$ & $\begin{array}{l}\text { A maior frequência na aulas práticas } \\
\text { está associada ao melhor desempenho } \\
\text { nas avaliações, não importando o } \\
\text { método de ensino. }\end{array}$ \\
\hline Han et al., 2015 & $\begin{array}{c}\text { Hospital da } \\
\text { Universidade Nacional } \\
\text { de Chungnam, Coréia } \\
\text { do Sul }\end{array}$ & $\begin{array}{l}134 \text { estudantes de } \\
\text { medicina }\end{array}$ & $\begin{array}{l}\text { Aulas práticas com observação } \\
\text { da dissecação ou práticas } \\
\text { realmente dissecando e depois } \\
\text { realizando tutoria. }\end{array}$ & $\begin{array}{l}\text { Os alunos que dissecavam e tutoravam } \\
\text { compreendiam melhor o conteúdo e } \\
\text { melhor desempenho nas avaliações. }\end{array}$ \\
\hline $\begin{array}{l}\text { Bergman et al., } \\
2015\end{array}$ & $\begin{array}{l}\text { Centro Médico da } \\
\text { Universidade Radboud, } \\
\text { Holanda }\end{array}$ & $\begin{array}{l}145 \text { estudantes do } \\
\text { primeiro ano do curso } \\
\text { bacharelado em } \\
\text { medicina }\end{array}$ & $\begin{array}{l}\text { Estudantes de medicina } \\
\text { responderam um pré-teste com } \\
\text { metades das questões contendo } \\
\text { informações do paciente, uma } \\
\text { semana depois estudaram a } \\
\text { anatomia musculoesquelética } \\
\text { sem informação sobre o } \\
\text { paciente (grupo controle) e }\end{array}$ & $\begin{array}{l}\text { Os resultados mostram que os alunos } \\
\text { que estudaram com contexto não } \\
\text { tiveram um desempenho melhor do } \\
\text { que os alunos que estudaram sem } \\
\text { contexto. Esta descoberta pode ser } \\
\text { explicada por uma interação do nível } \\
\text { de experiência dos participantes, a } \\
\text { natureza do conhecimento anatômico }\end{array}$ \\
\hline
\end{tabular}




\begin{tabular}{|c|c|c|c|c|}
\hline & & & $\begin{array}{l}\text { com informações (i) relevantes } \\
\text { sobre o paciente (grupo } \\
\text { experimental) e responderam } \\
\text { um pós teste. }\end{array}$ & $\begin{array}{l}\text { e as abordagens dos alunos para a } \\
\text { aprendizagem. }\end{array}$ \\
\hline Li \& Li, 2015 & $\begin{array}{l}\text { Binzhou Medical } \\
\text { University, China }\end{array}$ & 40 alunos de medicina & $\begin{array}{l}\text { Utilizando um exames de um } \\
\text { paciente ortopédico: } \\
\text { - Tomografia computadorizada } \\
\text { em duas dimensões; } \\
\text { - Imagens tridimensionais; } \\
\text { - Modelos anatômicos } \\
\text { tridimensionais (3Dp). }\end{array}$ & $\begin{array}{l}\text { O modelo anatômico ( } 3 \mathrm{Dp} \text { ) melhorou } \\
\text { notavelmente a identificação } \\
\text { anatômica da fratura da coluna } \\
\text { vertebral por estudantes de medicina. }\end{array}$ \\
\hline $\begin{array}{l}\text { Jones \& } \\
\text { Seckeler, } 2017\end{array}$ & $\begin{array}{c}\text { Faculdade de Medicina } \\
\text { da Universidade do } \\
\text { Arizona, EUA }\end{array}$ & Residentes médicos & $\begin{array}{l}\text { Pacientes com arcos aórticos } \\
\text { normais e anormais foram } \\
\text { selecionados e anonimizados } \\
\text { para gerar modelos impressos } \\
\text { em 3D, estes palestra de } 20 \\
\text { minutos sobre anéis vasculares } \\
\text { e fundas. Durante a palestra do } \\
\text { grupo de intervenção, modelos } \\
\text { físicos impressos em 3D de } \\
\text { cada lesão foram distribuídos } \\
\text { para inspeção. }\end{array}$ & $\begin{array}{l}\text { Um ganho mensurável no } \\
\text { conhecimento sobre anéis vasculares e } \\
\text { lingas de artéria pulmonar com a } \\
\text { adição de modelos impressos em 3D } \\
\text { dos defeitos. }\end{array}$ \\
\hline Akle et al., 2018 & $\begin{array}{l}\text { Universidade dos } \\
\text { Andes, Colômbia }\end{array}$ & $\begin{array}{l}\text { Estudantes de } \\
\text { medicina }\end{array}$ & $\begin{array}{l}\text { Construção de um modelo 3D a } \\
\text { partir de argila sobre as } \\
\text { estruturas periventriculares do } \\
\text { cérebro a partir de um vídeo } \\
\text { instrutivo. }\end{array}$ & $\begin{array}{l}\text { Os alunos que construíram os modelos } \\
\text { apresentaram pontuações maiores no } \\
\text { pós-teste do que aquelas ensinadas no } \\
\text { material de maneira mais tradicional, } \\
\text { além disso, a atividade de modelagem } \\
\text { reduziu o tempo gasto no estudo do } \\
\text { tópico e aumentou a compreensão das } \\
\text { relações espaciais entre estruturas no } \\
\text { cérebro. }\end{array}$ \\
\hline $\begin{array}{l}\text { Ladage et al., } \\
2018\end{array}$ & $\begin{array}{l}\text { Universidade Estadual } \\
\text { da Pensilvânia, EUA }\end{array}$ & $\begin{array}{l}\text { Estudantes recrutados } \\
\text { em cursos de } \\
\text { graduação em } \\
\text { psicologia e biologia }\end{array}$ & $\begin{array}{l}\text { Material sobre as conexões } \\
\text { estruturais da via visual e como } \\
\text { os danos ao longo das vias } \\
\text { estruturais podem afetar } \\
\text { diferencialmente o campo } \\
\text { visual utilizando material } \\
\text { elétricos. }\end{array}$ & $\begin{array}{l}\text { Ganhos de curto prazo podem ser } \\
\text { realizados com atividades de } \\
\text { aprendizado construtivas e ativas com } \\
\text { a utilização do modelo; no entanto, } \\
\text { não se encontrou diferenças nos } \\
\text { ganhos de aprendizado a longo prazo. }\end{array}$ \\
\hline
\end{tabular}

Fonte: Autores.

O uso da dissecação cadavérica é considerável fundamental no processo de aprendizado da anatomia, sendo incapaz de ser substituído por outros métodos (literatura e/ou informática), pois proporciona diferentes experiências tanto intelectual quanto educacional e emotiva. Além disso, o conhecimento anatômico é indispensável para a prática cirúrgica e segundo as Diretrizes Curriculares Nacionais do Curso de graduação em Medicina, presente na Resolução CNE/CES N 4 , de 7 de novembro de 2001, diz que, um dos objetivos da formação profissional do médico é proporcionar conhecimentos para que ele consiga desenvolver procedimentos clínicos e cirúrgicos essenciais no atendimento ambulatorial e nas urgências e emergências (Bernardes, 2015; Brasil, 2001). Certamente, isso explica a metodologia de ensino em medicina ser mais tradicional devido ao possível aspecto cirúrgico da profissão, enquanto as metodologias alternativas seriam maiores em outros cursos de saúde.

As publicações analisadas apresentam variedade de métodos de ensino. Todas as atividades propostas na metodologia e analisadas pelo estudo foram desenvolvidas em grupos discentes. Os tipos de métodos de ensino foram ativos $(\mathrm{n}=11$; $42,3 \%)$, passivos $(n=5 ; 19,2 \%)$ e um $(3,8 \%)$ que contemplava métodos ativos e passivos. As estratégias metodológicas propostas de forma única contemplavam cinco publicações, sendo as atividades: peça teatral, monitoria para a população, tapete em cavalo vivo, imagens, exame clínico por meio da anatomia palpatória e modelos anatômicos em argila. As atividades propostas como estratégias metodológicas foram organizadas, predominantemente, em mais de uma atividade comparadas entre si $(n=12 ; 46,2 \%)$. Desses estudos, oito utilizavam a dissecação cadavérica, quatro recursos tecnológicos, três modelos 
de plástico, dois trabalhos manuais, dois exames de imagem, dois recursos 2D, uma imagem em 3D, um estudo de prossecção, um com oficinas variadas, um de tutoria e um com pé do equino em impressora 3D.

As estratégias metodológicas utilizadas pelos artigos analisados foram satisfatórias para o processo ensinoaprendizagem discente. Quando avaliados individualmente, as peças teatrais, a tutoria de ensino, o tapete em cavalo vivo e os exames físicos utilizando a anatomia de superfície mostraram resultados positivos para a construção de um aprendizado significativo. Essas estratégias de ensino representam metodologias ativas no processo de ensino-aprendizado que tem como base o uso de problemáticas na construção desse conhecimento. É necessário mudar a metodologia ensinar-memorizar e partir para uma abordagem em que o discente assuma um papel mais ativo, construindo seu conhecimento por meio de buscas de soluções para problemas que surgem diante de si, sendo isso possível com uso de metodologias ativas. Visto que, existe uma complexidade no binômio ensino-aprendizagem devido à construção do conhecimento ser de forma dinâmica (Mitre et al., 2008).

A dissecação cadavérica humana, apesar de ser um método tradicional, demonstrou importância para a aquisição do conhecimento em Anatomia. Esta foi uma estratégia bastante utilizada para ser comparada as metodologias alternativas de ensino. As quatro publicações que compararam métodos de ensino variados com a dissecação cadavérica humana observaram que em 75\% ( $\mathrm{n}=03)$ a dissecação foi o melhor método quando comparada a trabalhos manuais, mídias virtuais, imagens tridimensionais e modelos plásticos. A dissecação cadavérica ainda possui um grande papel na ciência pois, ela proporciona a vivencia de momentos singulares para o desenvolvimento da conduta profissional (Pontinha \& Soeiro, 2014). A dissecação animal não demonstrou ser tão eficiente para o aprendizado da anatomia humana devido à diferença entre as espécies.

A impressão 3D foi estudada por quatro artigos, demonstrando ser melhor que a dissecação cadavérica humana, modelos virtuais, imagem em 2D e imagem tridimensional. Ter a tecnologia como aliada no ensino de anatomia humana proporciona resolução de problemas (financeiros, éticos, jurídicos e culturais) que surgem durante a obtenção de meios para realização deste ensino. Além disso, impressões 3D oferecem a oportunidade de observar estrutura grandes e estruturas de difíceis visualização, como ossos pequenos, cavidades, variações anatômicas e patologias (Matozinhos\& Madureira, 2017).

Trabalhos manuais foram estudados em dois artigos, obtendo um melhor desempenho quando comparados à dissecação animal e mídias virtuais. Um relato de experiência mostrou que o uso de massa de modelar e biscuit na confecção de corações sadios e com patologias no ensino de cardiologia pediátrica, proporcionou para um residente a possibilidade de melhor compreensão da anatomia do coração e localização de suas estruturas e alterações em exames de imagens, além disso, o documento mostra que é possível ser feito um bom trabalho na confecção desses materiais mesmo não tendo habilidades em artes plásticas, e intensifica a ideia de que mesmo com as diversas tecnologias existentes a arte ainda pode ser uma opção para auxiliar durante a formação (Amorim Junior\& Severi, 2018).

Os recursos virtuais, embora com resultados positivos, quando comparados à dissecação humana, trabalhos manuais e modelos de plástico, os discentes tiveram menor rendimento. $\mathrm{O}$ uso de tecnologias virtuais no ensino tem possibilitado benefícios para aprendizagem dos estudantes devido à sua facilidade, acessibilidade e flexibilidade em obter materiais para auxiliá-los, entretanto, mesmo com esses benefícios, as desvantagens no uso desse método estão relacionadas a parte técnica, como problemas com conexão à internet, hardware, software e outros mais que podem prejudicar o ensino (Wilcha, 2020).

\section{Considerações Finais}

Anatomia humana é uma ciência tradicional mantendo aspectos relevantes para sua evolução por meio da dissecação cadavérica. Porém, os estudos analisados demonstraram a importância da utilização de metodologias alternativas no processo de ensino aprendizagem. Os métodos de ensino de dissecação cadavérica e impressões 3D se destacaram quando comparadas 
com outras alterativas de ensino. E, quando avaliadas de forma individual, a dissecação cadavérica mostrou apresentar melhor resultado para aquisição do conhecimento.

Uma metodologia que não foi citada pelos artigos estudados, mas que pode ser uma ótima alternativa, é a utilização de jogos didático-pedagógicos para a construção do conhecimento, pois os jogos possibilitam melhorar a aprendizagem uma vez que ele proporciona a "oportunidade de desenvolver capacidades indispensáveis à sua futura formação e atuação profissional, tais como: atenção, afetividade, concentração e outras habilidades perceptuais psicomotoras” (Alves \& Bianchin, 2010, p. 3).

De modo geral, o uso de métodos alternativos mostrou ser de grande valia para o ensino da disciplina de anatomia humana, podendo então ser utilizados como estratégia auxiliar no ensino tradicional da disciplina. Por esse motivo, existe a necessidade de ampliar as pesquisas sobre metodologias alternativas para o ensino-aprendizagem em Anatomia Humana.

\section{Agradecimentos}

Os autores agradecem ao Programa de Fortalecimento Acadêmico (PFA) da UPE pelo apoio financeiro, uma vez que o presente trabalho recebeu financiamento por meio do Edital PROGRAD/PFAUPE/UPE 004/2019. Os autores também agradecem ao Prof. Me. Kleber Ferreira Costa, pela correção gramatical e ortográfica; às professoras Christiana Joy Maciel e. Deborah Joy Lopes, pela tradução do resumo em língua inglesa; ao professor Teilhard Masías Vergara, pela tradução do resumo em língua espanhola.

\section{Referências}

Akle, V., Peña-Silva, R. A., Valencia, D. M., \& Rincón-Perez, C. W. (2018). Validation of clay modeling as a learning tool for the periventricular structures of the human brain. Anatomical Sciences Education, 11(2): 137-145.

Alves, L., \& Bianchin, M. A. (2010). O jogo como recurso de aprendizagem. Revista Psicopedagogia, 27(83), $282-287$.

Amorim Junior, R. F. \& Severi, R. (2018). A arte no ensino da cardiologia: relato da experiência do uso de massas moldáveis no aprendizado da anatomia normal e patológica do coração. Revista Brasileira de Educação Médica, 42(4):103-108.

Barbosa, E. F. \& Moura, D. G. (2013). Metodologias ativas de aprendizagem na educação profissional e tecnológica. Boletim Técnico do Senac, 39 (2):48-67.

Bergman, E. M., \& Bruin, A. B. H. (2015). Effects of learning content in context on knowledge acquisition and recall: a pretest-posttest control group design. BMC Medical Education, 15 (2):133 - 134.

Bergman, E. M., Sieben, J. M. \& Smailbegovci. (2013). Constructive, collaborative, contextual, and self-directed learning in surface anatomy education. Anatomical Sciences Education, 6(2):114-24.

Bernardes, A. J. S. (2015). Anatomia e cirurgia. Revista Portuguesa de Cirurgia, (34):5-6.

Braid, F., \& Williams, S. B. (2012). Design and validation of a novel learning tool, the “Anato-Rug," for teaching equine topographical anatomy. Anatomical Sciences Education, 5(5):256-63.

Brasil (2001). Diretrizes Curriculares Nacionais do Curso de Graduação em Medicina. Resolução cne/ces nº 4, de 7 de novembro de 2001.

Brinke, B. T., \& Klitsie, P. J. (2014). Anatomy education and classroom versus laparoscopic dissection-based training: a randomized study at one medical school. Academic Medicine, 89 (5):806-810.

Codd, A. M., \& Choudhury, B. (2011). Virtual reality anatomy: is it comparable with traditional methods in the teaching of human forearm musculoskeletal anatomy? Anatomical Sciences Education, 4(3):119-25.

Costa, G., \& Costa, G. (2012). The corpse in the teaching of human anatomy: a methodological and bioethics overview. Revista Brasileira de Educação Médica, 39(3): 369-373.

Drake, R. L., \& Mcbride, J. M. (2009). Medical education in the anatomical sciences: the winds of change continue to blow. Anatomical Sciences Education, 2(6): 253-259.

Estevez, M. E., \& Lindgren, K. A. (2010). A novel three-dimensional tool for teaching human neuroanatomy. Anatomical Sciences Education; $3(6): 309-17$.

Fornaziero, C. C., \& Gordan, P. A. (2010). O ensino da anatomia: integração do corpo humano e meio ambiente. Revista Brasileira de Educação Médica, 34(2):290-297.

Garcia, F. (2013). A importância do uso das tecnologias no processo de ensino-aprendizagem. Educação a Distância, 3(1):25-48. 
Gonsalvez, D., Ovens, M. \& Ivanusic J. (2015). Does attendance at anatomy practical classes correlate with assessment outcome? A retrospective study of a large cohort of undergraduate anatomy students. BMC Medical Education, 15:231.

Gookin, J. L., \& Foster, D. M. (2009). An animated model of reticulorumen motility. Journal of Veterinary Medical Education, 36(4):444-447.

Han, E. R., Chung, E. K. \& Kwang-il nam, K. (2015). Peer-assisted learning in a gross anatomy dissection course. PLOS ONE, 13;10(11): :e0142988.

Haspel, C., \& Motoike, H. K. (2014). The implementation of clay modeling and rat dissection into the human anatomy and physiology curriculum of a large urban community college. Anatomical Sciences Education , 7(1):38-46.

Inzunza, O., \& D'acuña, E. (2003). Evaluación práctica de Anatomía. Rendimiento de los alumnos de primer año de medicina ante distintas formas de perguntar. International Journal of Morphology, 21(2):131-136.

Johnson, E., \& Charchanti, A. (2012). Modernization of an anatomy class: from conceptualization to implementation. A case for integrated multimodalmultidisciplinary teaching. Anatomical Sciences Education, 5(6):354-366.

Jones, T. W., \& Seckeler, M. D. (2017). Use of 3D models of vascular rings and slings to improve resident education. Congenital Heart Disease, 12(5):578582.

Khalil, M. K., Paas, F., Johnson, T. E., Su, Y. K., \& Payer, A. F. (2008). Effects of instructional strategies using cross sections on the recognition of anatomical structures in correlated CT and MR images. Anatomical Sciences Education, 1(2):75-83.

Keedy, A. W., Durack, J. C., Sandhu, P., Chen, E. M., O'sullivan, P. S. \& Breiman, R. S. (2011). Comparison of traditional methods with 3D computer models in the instruction of hepatobiliary anatomy. Anatomical Sciences Education, 4(2):84-91.

Kooloos, J. G. M., \& Schepens-Franke, A. N. (2014). Anatomical knowledge gain through a clay-modeling exercise compared to live and video observations. Anatomical Sciences Education, 7(6):420-429.

Knobe, M., \& Carow, J. B. (2012). Arthroscopy or ultrasound in undergraduate anatomy education: a randomized cross-over controlled trial. BMC Medical Education, 12:85.

Ladage, L. D., Tornello, S. L., Vallejera, J. M., Baker, E. E., Yan, Y., \& Chowdhury, A. (2018). Variation in behavioral engagement during an active learning activity leads to differential knowledge gains in college students. Advances in Physiology Education, 42(1):99-103.

Libâneo, J. C. (2013). Didática. 2. ed. São Paulo, SP: Cortez.

Li, Z., \& Li, Z. (2015). Three-dimensional printing models improve understanding of spinal fracture-a randomized controlled study in China. Scientific Reports, 5(1):11570.

Lombardi, S. A., \& Hicks, R. E. (2014). Are all hands-on activities equally effective? Effect of using plastic models, organ dissections, and virtual dissections on student learning and perceptions. Advances in Physiology Education, 38(1):80-86;

Masini, E. F. S., \& Moreira, M. A. (2008). Aprendizagem significativa: condições para ocorrência e lacunas que levam a comprometimentos. São Paulo, SP: Vetor.

Matozinhos, I. P., \& Madureira, A. A. C. (2017). Impressão 3D: inovações no campo da medicina. Revista Interdisciplinar Ciências Médicas, 1(1): 143-162.

Mclachlan, J. C., \& Bligh, J. (2004). Teaching anatomy without cadavers. Medical Education, 38(4): 418-424.

Mclachlan, J., \& Patten, D. (2006). Anatomy teaching: ghosts of the past, present and future. Medical Education, 40(3):243-253.

Miller, S. A., Perrotti, W., Silverthorn, D. U., Dalley, A. F. \& Rarey, K. E. (2002). From college to clinic: reasoning over memorization is key for understanding anatomy. The Anatomical Record, 269(2):69-80.

Mitre, S. M., Siqueira-Batista, R., Girardi-de-Mendonça, J. M., Morais-Pinto, N. M., Meirelles, C. A. B., Pinto-Porto, C., Moreira, T., \& Hoffmann, L. M. A. (2008). Metodologias ativas de ensino-aprendizagem na formação profissional em saúde: debates atuais. Ciência \& Saúde Coletiva, 13 (Suppl. 2), 2133-2144.

Montes, M. A. A., \& Souza, C. T. V. (2008). Atuação de alunos universitários em espaço não formal de ensino: estratégia motivadora e integradora para o desenvolvimento de atividades práticas e assistenciais. Alexandria: Revista de Educação em Ciência e Tecnologia, 1(2):63-79.

Montes, M. A. A., \& Souza, C. T. V. (2010). Estratégia de ensino-aprendizagem de anatomia humana para acadêmicos de medicina. Ciências \& Cognição, 15 (3):002-012.

Patel, K., \& Moxham, B. (2005) Attitudes of professional anatomists to curricular change. Clinical Anatomy, 19(2):132-141.

Pontinha, C. M., \& Soeiro, C. (2014). A dissecação como ferramenta pedagógica no ensino da Anatomia em Portugal. Interface - Comunicação Saúde Educação, 18(48):165-75.

Preece, D., \& Williams, S. B. (2013). "Let's Get Physical": advantages of a physical model over 3D computer models and textbooks in learning imaging anatomy. Anatomical Sciences Education, 6(4):216-224.

Ruisoto, P., \& Juanes, J. A. (2012). Experimental evidence for improved neuroimaging interpretation using three-dimensional graphic models. Anatomical Sciences Education, 5(3):132-137. 
Research, Society and Development, v. 10, n. 16, e370101623641, 2021

(CC BY 4.0) | ISSN 2525-3409 | DOI: http://dx.doi.org/10.33448/rsd-v10i16.23641

Salbego, C., \& Oliveira, E. M. D. (2015). Percepções acadêmicas sobre o ensino e a aprendizagem em anatomia humana. Revista Brasileira Educação Médica, 39(1):23-31.

Serrat, M. A., \& Dom, A. M. (2014). Independent learning modules enhance student performance and understanding of anatomy. Anatomical Sciences Education, 7(5):406-416

Severino, A. J. (2009). Pós-graduação e pesquisa: o processo de produção e de sistematização do conhecimento. Diálogo Educacional, 9(26): 13-27.

Silva A. V., \& Aguiar Junior, O. (2010). O ser humano em sua dimensão biológica: metodologias ativas de ensino e de avaliação na graduação em saúde em um contexto interprofissional. PBL 2010 Congresso Internacional. São Paulo, Brasil.

Silva, R. A., \& Silva, M. L. (2001). O ensino da anatomia através das artes cênicas. Arquivos da APADEC, 5(1):9-14.

Silva, K., \& Santana, O (2012). Objetos de aprendizagem utilizados para o ensino da anatomia humana: uma revisão de literatura. Anais...São Luís: ABED.

Soares, M. H. F. B. (2008). Jogos e atividades lúdicas no ensino de química: teoria, métodos e aplicações. Guarapari, ES: Ex Libris.

Waters, J. R., \& Meter, P. V. (2011). Human clay models versus cat dissection: how the similarity between the classroom and the exam affects student performance. Advances in Physiology Education, 35(2):227-236.

Wilcha, R-J. (2020). Effectiveness of virtual medical teaching during the COVID-19 crisis: systematic review. JMIR Medical Education, 6(2):e20963. 